\title{
The Second Kummer Function with Matrix Parameters and Its Asymptotic Behaviour
}

\author{
Georg Wehowar (i) and Erika Hausenblas \\ Montanuniversität Leoben, Austria \\ Correspondence should be addressed to Erika Hausenblas; erika.hausenblas@unileoben.ac.at
}

Received 17 April 2018; Revised 23 September 2018; Accepted 8 October 2018; Published 2 December 2018

Academic Editor: Lucas Jodar

Copyright (C) 2018 Georg Wehowar and Erika Hausenblas. This is an open access article distributed under the Creative Commons Attribution License, which permits unrestricted use, distribution, and reproduction in any medium, provided the original work is properly cited.

In the present article, we introduce the second Kummer function with matrix parameters and examine its asymptotic behaviour relying on the residue theorem. Further, we provide a closed form of a solution of a Weber matrix differential equation and give a representation using the second Kummer function.

\section{Introduction}

The application of special functions can be found in theoretical physics [1], probability theory $[1,2]$, or numerical mathematics [1]. The solution of the confluent hypergeometric differential equation [3] is often expressed as a linear combination of the Kummer functions that are defined as

$$
\begin{aligned}
& \mathrm{M}(a, b, z)=\sum_{k=0}^{\infty} \frac{(a)_{k}}{(b)_{k} k !} z^{k}, \\
& \mathrm{U}(a, b, z)=\frac{\pi}{\sin (\pi b)}\left(\frac{\mathrm{M}(a, b, z)}{\Gamma(1+a-b) \Gamma(b)}\right. \\
& \left.\quad-z^{1-b} \frac{\mathrm{M}(1+a-b, 2-b, z)}{\Gamma(a) \Gamma(2-b)}\right),
\end{aligned}
$$

where $z$ is a complex argument and $a$ and $b$ are real-valued parameters that are not negative integers. We note that $\mathrm{U}$ is also known as the second confluent hypergeometric, Tricomi, or Gordon function. Its asymptotic behaviour is well known (see [3]): in particular for $|z| \longrightarrow \infty$, we have

$$
\mathrm{U}(a, b, z)=z^{-a}\left(1+O\left(|z|^{-1}\right)\right) .
$$

The generalization of special functions to matrix valued functions is a growing subject and the first Kummer function has been studied widely (see [4-7]). However, the second Kummer function has not yet been examined.
The main goal of this article is to introduce the second Kummer function with matrix parameters and to study its asymptotic behaviour. This function appears as a solution of an equation in mathematical finance, where a Markovian regime switching framework (see $[8,9]$ as an example) is combined with an equilibrium model for asset bubbles from $[10,11]$. In such a model, knowing the asymptotic behaviour of the solution is essential.

Currently, there is a growing number of literatures about matrix special functions. The study of the properties of Gamma and Beta matrix functions by Jódar and Cortés [5] is a corner stone of the theory of matrix special functions and provides us with many important concepts to examine their properties. Moreover, Jódar and Cortés [6, 12] also later introduced the first Kummer matrix function, gave an integral representation, and used them to obtain a solution in a closed form of a hypergeometric matrix differential equation. For solving a matrix differential equations, matrix polynomials were studied frequently, such as the Laguerre matrix polynomials [13-15], the Hermite matrix polynomials [14], the Jacobi matrix polynomials [16], or the Gegenbauer matrix polynomials [17]. Many other matrix special functions were already introduced. The modified Gamma matrix and the incomplete Bessel function were studied in [18] and the Humbert matrix functions in $[19,20]$. A modification of the first Kummer matrix function including two complex variables was introduced in [7]. Recently, the hypergeometric 
matrix functions were extended by adding another matrix parameter (see [4]).

Throughout the paper, we will use the following notation. Let $\mathrm{L}$ and $\mathrm{M}$ be $N \times N$ matrices. With $\mathrm{I}$ we denote the identity matrix. Given a vector $v \in \mathbb{C}^{N}$, we use $\operatorname{diag}(v)$ for the matrix with $v$ in its diagonal entries and zero elsewhere. We call a matrix positive stable, if it has only eigenvalues with positive real part. For complex valued matrix functions $f$ and $g$, we write $f(z) \sim g(z)$ if there is a matrix C such that $\lim _{|z| \rightarrow \infty} f(z)=\lim _{|z| \rightarrow \infty} g(z)$ C. We write $\lfloor x\rfloor$ for the integer part of $x \in \mathbb{R}$. The symbol $\lesssim$ means asymptotically smaller.

The article is structured as follows. In Section 2, we repeat some of the most important concepts from matrix special function theory. It contains the definition of the second Kummer function, with matrix parameters $\mathrm{L}$ and $b \mathrm{I}$ and a complex argument $z$, as

$$
\begin{aligned}
\mathrm{U}(\mathrm{L}, b \mathrm{I}, z)=\Gamma & (1-b){ }_{1} F_{1}(\mathrm{~L} ; b \mathrm{I} ; z) \Gamma(\mathrm{L}+(1-b) \mathrm{I})^{-1} \\
+ & z^{1-b} \Gamma(b-1) \\
& \cdot{ }_{1} F_{1}((1-b) \mathrm{I}+\mathrm{L} ;(2-b) \mathrm{I} ; z) \\
& \cdot \Gamma(\mathrm{L})^{-1} .
\end{aligned}
$$

Based on a classical approach as in Slater [21] or Paris and Kaminski [22], we analyse the asymptotic behaviour of this function in Section 3. In particular, we show the asymptotic behaviour for large $|z|$,

$$
\mathrm{U}(\mathrm{L}, b \mathrm{I}, z) \sim|z|^{-\mathrm{L}}
$$

under certain conditions on the matrix L. Moreover, we introduce the parabolic cylinder function with matrix parameters in the present article and analyse its asymptotic behaviour. In Section 4, we compute a solution of a Weber matrix differential equation

$$
y^{\prime \prime}(x)+\left(\mathrm{K}+\left(\frac{1}{2}-\frac{x^{2}}{4}\right) \mathrm{I}\right) y(x)=0, \quad x \geq 0,
$$

using the power series method. The representation of this solution uses parabolic cylinder functions with matrix parameters.

\section{Some Examples of Special Matrix Functions}

First, we define the Pochhammer symbol for matrices as

$$
\begin{aligned}
& (\mathrm{M})_{k}=(\mathrm{M}+(k-1) \mathrm{I}) \cdots(\mathrm{M}+\mathrm{I}) \mathrm{M} \text { for } k \geq 1, \\
& (\mathrm{M})_{0}=\mathrm{I} .
\end{aligned}
$$

Using the matrix exponential, we define

$$
t^{\mathrm{M}}=e^{\mathrm{M} \ln t}=\sum_{k=0}^{\infty} \mathrm{M}^{k} \frac{(\ln t)^{k}}{k !}
$$

for $t>0$. Following [5], we introduce the Gamma matrix function for a positive stable matrix $\mathrm{M}$ as

$$
\Gamma(\mathrm{M})=\int_{0}^{\infty} e^{-t} t^{\mathrm{M}-\mathrm{I}} d t
$$

Using infinite matrix products [23], the Gamma matrix function can be extended to matrices with only nonnegativeinteger eigenvalues, i.e., $-n \notin \sigma(\mathrm{M})$ for $n \in \mathbb{N} \backslash\{0\}$. If $\mathrm{M}+n \mathrm{I}$ is an invertible matrix for every integer $n \geq 0$, then it can be shown that $\Gamma(\mathrm{M})$ is also invertible and its inverse corresponds to the inverse of the Gamma function (see [5]). Computing $\Gamma(\mathrm{M})$ numerically for a diagonalizable matrix $\mathrm{M}=\mathrm{TDT}^{-1}$ is simple, as we have

$$
\Gamma(\mathrm{M})=\Gamma\left(\mathrm{TDT}^{-1}\right)=\int_{0}^{\infty} e^{-t} \mathrm{~T} e^{\mathrm{D} \ln (t)} \mathrm{T}^{-1} t^{-1} d t=\mathrm{TGT}^{-1}
$$

where the matrix

$$
\mathrm{G}=\left(\begin{array}{cccc}
\Gamma\left(\mu_{1}\right) & 0 & \cdots & 0 \\
0 & \Gamma\left(\mu_{2}\right) & \cdots & 0 \\
\vdots & \vdots & \ddots & \vdots \\
0 & 0 & \cdots & \Gamma\left(\mu_{N}\right)
\end{array}\right)
$$

contains Gamma functions of eigenvalues the $\mu_{1}, \ldots, \mu_{N}$ of $\mathrm{M}$.

Now we define the Beta matrix function for positive stable matrices $\mathrm{L}$ and $\mathrm{M}$ as

$$
B(\mathrm{~L}, \mathrm{M})=\int_{0}^{1} t^{\mathrm{L}-\mathrm{I}}(1-t)^{\mathrm{M}-\mathrm{I}} d t .
$$

This function is symmetric if and only if $L$ and $M$ commute [5]. The next lemma (see Lemma 2 from [12]) characterizes the relationship between Beta and Gamma matrix function.

Lemma 1. For positive stable, commuting matrices $\mathrm{L}$ and $\mathrm{M}$ so that $\mathrm{L}+\mathrm{M}$ has only nonnegative-integer eigenvalues, the following holds:

$$
B(\mathrm{~L}, \mathrm{M})=\Gamma(\mathrm{L}) \Gamma(\mathrm{M}) \Gamma(\mathrm{L}+\mathrm{M})^{-1} .
$$

Proof. First, we write

$$
\begin{aligned}
\Gamma(\mathrm{L}) \Gamma(\mathrm{M}) & =\left(\int_{0}^{\infty} e^{-s} s^{\mathrm{L}-\mathrm{I}} d s\right)\left(\int_{0}^{\infty} e^{-t} t^{\mathrm{M}-\mathrm{I}} d t\right) \\
& =\int_{0}^{\infty} \int_{0}^{\infty} e^{-s} s^{\mathrm{L}-\mathrm{I}} e^{-t} t^{\mathrm{M}-\mathrm{I}} d s d t .
\end{aligned}
$$

With the change of variables $x=s /(s+t)$ and $y=s+t$ and using the commutativity, we get

$$
\begin{aligned}
\Gamma(\mathrm{L}) & \Gamma(\mathrm{M})=\int_{0}^{\infty} \int_{0}^{1} e^{-x y}(x y)^{\mathrm{L}-\mathrm{I}} \\
\cdot & e^{-y(1-x)}(y(1-x))^{\mathrm{M}-\mathrm{I}} y d x d y . \\
= & \left(\int_{0}^{\infty} e^{-y} y^{\mathrm{L}+\mathrm{M}-\mathrm{I}} d y\right)\left(\int_{0}^{1} x^{\mathrm{L}-\mathrm{I}}(x-1)^{\mathrm{M}-\mathrm{I}} d y\right) \\
= & \Gamma(\mathrm{L}+\mathrm{M}) B(\mathrm{~L}, \mathrm{M}) .
\end{aligned}
$$

Due to the extension of the Gamma function [23], we do not need the additional condition from Lemma 2 in [12] that $L+M$ has to be positive stable. Since $\Gamma(\mathrm{L}+\mathrm{M})$ is well-defined, it is invertible and we obtain the desired result. 
The following lemma taken from [12] gives us an integral representation of the Pochhammer matrix symbol.

Lemma 2. Let $\mathrm{L}, \mathrm{M}$ and $\mathrm{M}-\mathrm{L}$ be positive stable matrices such that LM = ML. Then the following identity holds:

$$
\begin{aligned}
(\mathrm{L})_{k}(\mathrm{M})_{k}^{-1}= & \Gamma(\mathrm{L})^{-1} \Gamma(\mathrm{M}-\mathrm{L})^{-1} \\
& \cdot\left(\int_{0}^{1} t^{\mathrm{L}+(k-1) \mathrm{I}}(1-t)^{\mathrm{M}-\mathrm{L}-\mathrm{I}} d t\right) \Gamma(\mathrm{M}),
\end{aligned}
$$

for every $k \in \mathbb{N}$.

Proof. Using Lemma 1 and the fact that L and M commute, we get

$$
\begin{aligned}
(\mathrm{L})_{k}(\mathrm{M})_{k}^{-1}=\Gamma(\mathrm{L})^{-1} \Gamma(\mathrm{L}+k \mathrm{I}) \Gamma(\mathrm{M}) \Gamma(\mathrm{M}+k \mathrm{I})^{-1} \\
=\Gamma(\mathrm{L})^{-1} \Gamma(\mathrm{L}+k \mathrm{I}) \Gamma(\mathrm{M}+k \mathrm{I})^{-1} \Gamma(\mathrm{M})=\Gamma(\mathrm{L})^{-1} \\
\cdot \Gamma(\mathrm{M}-\mathrm{L})^{-1} \Gamma(\mathrm{M}-\mathrm{L}) \Gamma(\mathrm{L}+k \mathrm{I}) \Gamma(\mathrm{M}+k \mathrm{I})^{-1} \Gamma(\mathrm{M}) \\
=\Gamma(\mathrm{L})^{-1} \Gamma(\mathrm{M}-\mathrm{L})^{-1} B(\mathrm{~L}+k \mathrm{I}, \mathrm{M}-\mathrm{L}) \Gamma(\mathrm{M}) \\
=\Gamma(\mathrm{L})^{-1} \Gamma(\mathrm{M}-\mathrm{L})^{-1}\left(\int_{0}^{1} t^{\mathrm{L}+(k-1) \mathrm{I}}(1-t)^{\mathrm{M}-\mathrm{L}-\mathrm{I}} d t\right) \\
\cdot \Gamma(\mathrm{M}) .
\end{aligned}
$$

We remark that the Beta function is well-defined, because $\mathrm{M}$ $\mathrm{L}$ is positive stable.

We define the confluent hypergeometric function with matrix parameters as

$$
\left.{ }_{1} F_{1}(\mathrm{~L} ; \mathrm{M} ; z)=\sum_{k=0}^{\infty}(\mathrm{L}){ }_{k}(\mathrm{M})\right)_{k}^{-1} \frac{z^{k}}{k !}
$$

for $z \in \mathbb{C}$, where $(\mathrm{M}+k \mathrm{I}$ ) invertible for every $k \geq 0$ (see [15]). This function is also often called first Kummer function and the notation $\mathrm{M}(\mathrm{L}, \mathrm{M}, z)={ }_{1} F_{1}(\mathrm{~L} ; \mathrm{M} ; z)$ can be found elsewhere. Now let $\mathrm{L}$ and $\mathrm{M}$ be commuting matrices. We obtain the integral representation.

Lemma 3. If $\mathrm{M}-\mathrm{L}$ is positive stable, then we have

$$
\begin{aligned}
{ }_{1} F_{1}(\mathrm{~L} ; \mathrm{M} ; z)= & \Gamma(\mathrm{L})^{-1} \Gamma(\mathrm{M}-\mathrm{L})^{-1} \\
& \cdot\left(\int_{0}^{1} e^{z t} t^{\mathrm{L}-\mathrm{I}}(1-t)^{\mathrm{M}-\mathrm{L}-\mathrm{I}} d t\right) \Gamma(\mathrm{M})
\end{aligned}
$$

for all $z \in \mathbb{C}$.

Proof. By Lemma 2, we get

$$
\begin{aligned}
& { }_{1} F_{1}(\mathrm{~L} ; \mathrm{M} ; z)=\sum_{k=0}^{\infty} \Gamma(\mathrm{L})^{-1} \Gamma(\mathrm{M}-\mathrm{L})^{-1} \\
& \quad\left(\int_{0}^{1} t^{\mathrm{L}+(k-1) \mathrm{I}}(1-t)^{\mathrm{M}-\mathrm{L}-\mathrm{I}} d t\right) \Gamma(\mathrm{M}) \frac{z^{k}}{k !}=\Gamma(\mathrm{L})^{-1}
\end{aligned}
$$

$\cdot \Gamma(\mathrm{M}-\mathrm{L})^{-1}\left(\int_{0}^{1} t^{\mathrm{L}-\mathrm{I}} \sum_{k=0}^{\infty} \frac{(t z)^{k}}{k !}(1-t)^{\mathrm{M}-\mathrm{L}-\mathrm{I}} d t\right)$

$\cdot \Gamma(\mathrm{M})$.

If $L$ commutes with $M$, it consequently commutes with ( $M+$ $k \mathrm{I})^{-1}$ for all integers $k \geq 0$ and we get

$$
\begin{aligned}
\frac{d}{d z}{ }_{1} F_{1}(\mathrm{~L} ; \mathrm{M} ; z) & =\sum_{k=1}^{\infty}(\mathrm{L})_{k}(\mathrm{M})_{k}^{-1} \frac{z^{k-1}}{(k-1) !} \\
& =\sum_{k=0}^{\infty}(\mathrm{L})_{k+1}(\mathrm{M})_{k+1}^{-1} \frac{z^{k}}{k !} \\
& =\sum_{k=0}^{\infty}(\mathrm{L}+\mathrm{I})_{k} \mathrm{~L}(\mathrm{M}+\mathrm{I})_{k}^{-1} \mathrm{M}^{-1} \frac{z^{k}}{k !} \\
& =\left(\sum_{k=0}^{\infty}(\mathrm{L}+\mathrm{I})_{k}(\mathrm{M}+\mathrm{I})_{k}^{-1} \frac{z^{k}}{k !}\right) \mathrm{LM}^{-1} \\
& ={ }_{1} F_{1}(\mathrm{~L}+\mathrm{I} ; \mathrm{M}+\mathrm{I} ; z) \mathrm{LM}^{-1} .
\end{aligned}
$$

Since $(\mathrm{L}+k \mathrm{I})$ commutes with $(\mathrm{M}+k \mathrm{I})^{-1}$ for all integers $k \geq 0$, we obtain

$$
\frac{d^{k}}{d z^{k}}{ }_{1} F_{1}(\mathrm{~L} ; \mathrm{M} ; z)={ }_{1} F_{1}(\mathrm{~L}+k \mathrm{I} ; \mathrm{M}+k \mathrm{I} ; z)(\mathrm{L}){ }_{k}(\mathrm{M})_{k}^{-1} .
$$

Note that this property holds especially for diagonal matrices M. We define the second Kummer function with matrix parameters as

$$
\begin{aligned}
\mathrm{U}(\mathrm{L}, b \mathrm{I}, z)= & \Gamma(1-b){ }_{1} F_{1}(\mathrm{~L} ; b \mathrm{I} ; z) \\
& \cdot \Gamma(\mathrm{L}+(1-b) \mathrm{I})^{-1}+z^{1-b} \Gamma(b-1) \\
& \cdot{ }_{1} F_{1}((1-b) \mathrm{I}+\mathrm{L} ;(2-b) \mathrm{I} ; z) \\
& \cdot \Gamma(\mathrm{L})^{-1}
\end{aligned}
$$

for $z \in \mathbb{C}$, where $b \in \mathbb{R} \backslash \mathbb{Z}^{-}$. Moreover, we introduce the matrix function

$$
\begin{aligned}
\mathrm{F}_{\mathrm{K}}(z)= & \Gamma\left(\frac{1}{2}\right){ }_{1} F_{1}\left(-\frac{1}{2} \mathrm{~K} ; \frac{1}{2} \mathrm{I} ; \frac{z^{2}}{2}\right) \Gamma\left(\frac{1}{2}(\mathrm{I}-\mathrm{K})\right)^{-1} \\
+ & \frac{|z| \Gamma(-1 / 2)}{\sqrt{2}}{ }_{1} F_{1}\left(\frac{1}{2}(\mathrm{I}-\mathrm{K}) ; \frac{3}{2} \mathrm{I} ; \frac{z^{2}}{2}\right) \\
& \cdot \Gamma\left(-\frac{1}{2} \mathrm{~K}\right)^{-1},
\end{aligned}
$$

for $z \in \mathbb{C}$ and matrices $\mathrm{K}$ having only eigenvalues with negative real part. Obviously, $\mathrm{F}_{\mathrm{K}}(z)=\mathrm{U}\left(-(1 / 2) \mathrm{K},(1 / 2) \mathrm{I}, z^{2} / 2\right)$. Analogously to the definition in $[24$, p. 39], we call

$$
\mathrm{D}_{\mathrm{K}}(z)=2^{\mathrm{K} / 2} e^{-z^{2} / 4} \mathrm{~F}_{\mathrm{K}}(z)
$$


the parabolic cylinder function with matrix parameters. We remark that

$$
\begin{aligned}
\frac{d}{d z} \mathrm{~F}_{\mathrm{K}}(z)=\Gamma\left(\frac{1}{2}\right){ }_{1} F_{1}\left(\frac{1}{2}(2 \mathrm{I}-\mathrm{K}) ; \frac{3}{2} \mathrm{I} ; \frac{z^{2}}{2}\right)(-\mathrm{K}) \\
\cdot \Gamma\left(\frac{1}{2}(\mathrm{I}-\mathrm{K})\right)^{-1}+\frac{\Gamma(-1 / 2)}{\sqrt{2}} \\
\cdot{ }_{1} F_{1}\left(\frac{1}{2}(\mathrm{I}-\mathrm{K}) ; \frac{3}{2} \mathrm{I} ; \frac{z^{2}}{2}\right) \\
\cdot \Gamma\left(-\frac{1}{2} \mathrm{~K}\right)^{-1} \operatorname{sgn}(z)+\frac{\Gamma(-1 / 2)}{\sqrt{2}} \\
\cdot{ }_{1} F_{1}\left(\frac{1}{2}(3 \mathrm{I}-\mathrm{K}) ; \frac{5}{2} \mathrm{I} ; \frac{z^{2}}{2}\right) \frac{1}{3}(\mathrm{~K}-\mathrm{I}) \\
\cdot \Gamma\left(-\frac{1}{2} \mathrm{~K}\right)^{-1} z^{2},
\end{aligned}
$$

for $z \in \mathbb{R} \backslash\{0\}$. In $z=0$ the function $\mathrm{F}_{\mathrm{K}}(z)$ is obviously not differentiable; however we can observe that

$$
\begin{aligned}
& \left.\partial_{+} \mathrm{F}_{\mathrm{K}}(z)\right|_{z=0}=\frac{\Gamma(-1 / 2)}{\sqrt{2}} \Gamma\left(-\frac{1}{2} \mathrm{~K}\right)^{-1}, \\
& \left.\partial_{-} \mathrm{F}_{\mathrm{K}}(z)\right|_{z=0}=-\frac{\Gamma(-1 / 2)}{\sqrt{2}} \Gamma\left(-\frac{1}{2} \mathrm{~K}\right)^{-1} .
\end{aligned}
$$

\section{Asymptotic Behaviour of the Second Kummer Function}

Now we focus on the asymptotic behaviour of the second Kummer function with matrix parameters. Analogously to the case with real-valued parameters (see [21, p. 35] or [22, p. 106]), we compute the Mellin-Barnes integral using the residue theorem. The proof of the following lemma has two steps. First, we define integral over a curve depending on $R>0$ and apply the residue theorem. The sum of the residues converges to an expression containing the second Kummer function. Then, by parametrizing the curve and taking limits, we obtain another representation for the integral.

Lemma 4. Let $\mathrm{L}$ be a positive stable, diagonalizable matrix and $b \in \mathbb{R} \backslash \mathbb{Z}^{-}$. For $z \in \mathbb{C}$ with $|\arg (z)|<3 \pi / 2$ and $c<\infty$, the following holds:

$$
\begin{aligned}
& \frac{1}{2 \pi i} \int_{\mathcal{c}-i \infty}^{c+i \infty} \Gamma(-s) \Gamma(\mathrm{L}+s \mathrm{I}) \Gamma(\mathrm{L}+(1-b+s) \mathrm{I}) \\
& \quad \cdot|z|^{-s} d s=|z|^{\mathrm{L}} \mathrm{U}(\mathrm{L}, b \mathrm{I}, z) \Gamma(\mathrm{L}) \Gamma(\mathrm{L}+(1-b) \mathrm{I}) .
\end{aligned}
$$

Proof. Let $\lambda_{1}, \ldots, \lambda_{N}$ denote the eigenvalues of $\mathrm{L}$ and suppose we have the eigenvalue decomposition $\mathrm{L}=\mathrm{T} \Lambda \mathrm{T}^{-1}$ where $\Lambda=$ $\operatorname{diag}\left(\left(\lambda_{1}, \ldots, \lambda_{N}\right)^{\top}\right)$. Since $\mathrm{L}$ is positive stable, all eigenvalues have nonnegative real part and, hence, all singularities of
$\Gamma\left(\lambda_{i}+s\right)$ are on the negative real axis. Let $\alpha, \beta$ and $R$ be positive real numbers. For each eigenvalue, let us consider the integral

$$
\begin{aligned}
& \Im_{\lambda_{i}, R}=\frac{1}{2 \pi i} \oint_{\mathscr{C}_{\lambda_{i}}} \Gamma(-s) \Gamma\left(\lambda_{i}+s I\right) \Gamma\left(\lambda_{i}+(1-b+s)\right) \\
& \cdot|z|^{-s} d s,
\end{aligned}
$$

where $\mathscr{C}_{\lambda_{i}}$ is taken around a rectangular contour so that the poles at $s=-\lambda_{i}-k$ and at $s=-\lambda_{i}-(1-b+k)$ are inside and all other poles are outside the contour for all $i \in 1, \ldots, N$ and $k=0,1,2, \ldots,\lfloor R\rfloor$. So, according to the residue theorem, we get

$$
\begin{aligned}
& \Im_{\lambda_{i}, R}=|z|^{\lambda_{i}} \sum_{k=0}^{\lfloor R\rfloor} \Gamma\left(\lambda_{i}+k\right) \Gamma(1-b-k) \frac{(-|z|)^{k}}{k !} \\
& +|z|^{\lambda_{i}}|z|^{1-b} \\
& \quad \cdot \sum_{k=0}^{\lfloor R\rfloor} \Gamma\left(\lambda_{i}+1-b+k\right) \Gamma(b-(k+1)) \frac{(-|z|)^{k}}{k !} .
\end{aligned}
$$

We remark that $\mathrm{L}+k \mathrm{I}$ has the eigenvalue decomposition $\mathrm{T}(\Lambda+$ $k I) \mathrm{T}^{-1}$. Defining the matrix

$$
\mathfrak{J}_{R}=\mathrm{T} \operatorname{diag}\left(\left(\mathfrak{\Im}_{\lambda_{1}, R}, \ldots, \mathfrak{\Im}_{\lambda_{N}, R}\right)^{\top}\right) \mathrm{T}^{-1}
$$

we can write

$$
\begin{aligned}
\Im_{R} & =|z|^{\mathrm{L}} \sum_{k=0}^{\lfloor R\rfloor} \Gamma(\mathrm{L}+k \mathrm{I}) \Gamma(1-b-k) \frac{(-|z|)^{k}}{k !}+|z|^{\mathrm{L}} \\
& \cdot|z|^{1-b} \\
& \cdot \sum_{k=0}^{\lfloor R\rfloor} \Gamma(\mathrm{L}+(1-b+k) \mathrm{I}) \Gamma(b-(k+1)) \frac{(-|z|)^{k}}{k !} .
\end{aligned}
$$

In the next step, we use the relationship $\Gamma(\mathrm{L}+k \mathrm{I})=(\mathrm{L})_{k} \Gamma(\mathrm{L})$ for Gamma matrix functions and the identity

$$
(-1)^{k} \Gamma(1-b-k)=\frac{\Gamma(1-b)}{(b)_{k}}
$$

for Gamma functions. We denote $\mathfrak{J}=\lim _{R \rightarrow \infty} \mathfrak{J}_{R}$ and obtain

$$
\begin{aligned}
\mathfrak{I} & =|z|^{\mathrm{L}} \sum_{k=0}^{\infty} \frac{(\mathrm{L})_{k}}{(b)_{k}} \frac{|z|^{k}}{k !} \Gamma(\mathrm{L}) \Gamma(1-b)+|z|^{\mathrm{L}}|z|^{1-b} \\
& \cdot \sum_{k=0}^{\infty} \frac{(\mathrm{L}+(1-b) \mathrm{I})_{k}}{(2-b)_{k}} \frac{|z|^{k}}{k !} \Gamma(\mathrm{L}+(1-b) \mathrm{I}) \Gamma(b-1) \\
& =|z|^{\mathrm{L}}{ }_{1} F_{1}(\mathrm{~L} ; b \mathrm{I} ; z) \Gamma(\mathrm{L}) \Gamma(1-b)+|z|^{\mathrm{L}}|z|^{1-b} \\
& \cdot{ }_{1} F_{1}(\mathrm{~L}+(1-b) \mathrm{I} ;(2-b) \mathrm{I} ; z) \Gamma(\mathrm{L}+(1-b) \mathrm{I}) \\
& \cdot \Gamma(b-1) .
\end{aligned}
$$


As $\mathrm{L}$ and $\mathrm{L}+(1-b)$ I commute, also the matrix exponential and hence their Gamma matrix function commute. Therefore, we get

$$
\mathfrak{I}=|z|^{\mathrm{L}} \mathrm{U}(\mathrm{L}, b \mathrm{I}, z) \Gamma(\mathrm{L}) \Gamma(\mathrm{L}+(1-b) \mathrm{I}) .
$$

Now we want to find an integral representation for $\mathfrak{\Im}$. Therefore, we examine the contour $\mathscr{C}_{\lambda_{i}}$ for $R \longrightarrow \infty$ for each eigenvalue $\lambda_{i}$. Hence, we parametrize the contour and write the integral as

$$
\mathfrak{\Im}_{\lambda_{i}, R}=\mathfrak{I}_{\lambda_{i}, \mathscr{C}_{1}}+\mathfrak{\Im}_{\lambda_{i}, \mathscr{C}_{2}}+\mathfrak{\Im}_{\lambda_{i}, \mathscr{C}_{3}}+\mathfrak{\Im}_{\lambda_{i}, \mathscr{C}_{4}},
$$

where we use the abbreviations

$$
\begin{aligned}
& \Im_{\lambda_{i}, \mathscr{C}_{1}}=\frac{1}{2 \pi i} \int_{c}^{-R} \Gamma(-x-i \alpha) \Gamma\left(\lambda_{i}+x+i \alpha\right) \\
& \cdot \Gamma\left(\lambda_{i}+1-b+x+i \alpha\right)|z|^{-x-i \alpha} d x \\
& \mathfrak{J}_{\lambda_{i}, \mathscr{C}_{2}}=-\frac{1}{2 \pi i} \int_{c-i \beta}^{c+i \alpha} \Gamma(R-t) \Gamma\left(\lambda_{i}-R+t\right) \\
& \cdot \Gamma\left(\lambda_{i}+1-b-R+t\right)|z|^{R-t} d t \\
& \mathfrak{\Im}_{\lambda_{i}, \mathscr{C}_{3}}=\frac{1}{2 \pi i} \int_{-R}^{c} \Gamma(-x+i \beta) \Gamma\left(\lambda_{i}+x-i \beta\right) \\
& \cdot \Gamma\left(\lambda_{i}+1-b+x-i \beta\right)|z|^{-x+i \beta} d x, \\
& \mathfrak{\Im}_{\lambda_{i}, \mathscr{C}_{4}}=\frac{1}{2 \pi i} \int_{c-i \beta}^{c+i \alpha} \Gamma(-s) \Gamma\left(\lambda_{i}+s\right) \Gamma\left(\lambda_{i}+1-b+s\right) \\
& \cdot|z|^{-s} d s .
\end{aligned}
$$

In the next step, we show that $\mathfrak{\Im}_{\lambda_{i}, \mathscr{C}_{1}} \longrightarrow 0$ and $\mathfrak{\Im}_{\lambda_{i}, \mathscr{C}_{3}} \longrightarrow 0$. Following [21], we use the Stirling formula

$$
|\Gamma(u+i v)| \lesssim \sqrt{2 \pi}|v|^{u-1 / 2} e^{-(\pi / 2)|v|}
$$

for the Gamma function with $|u|$ finite and $|v|$ large (see [25, p. 223]). Altogether, we get

$$
\begin{aligned}
& |\Gamma(-x-i \alpha)| \lesssim \sqrt{2 \pi} \alpha^{-x-1 / 2} e^{-(\pi / 2) \alpha}, \\
& \left|\Gamma\left(\lambda_{i}+x+i \alpha\right)\right| \\
& \quad \lesssim \sqrt{2 \pi}\left(\alpha+\operatorname{Im}\left(\lambda_{i}\right)\right)^{\operatorname{Re}\left(\lambda_{i}\right)+x-1 / 2} e^{-(\pi / 2)\left(\alpha+\operatorname{Im}\left(\lambda_{i}\right)\right)}, \\
& \left|\Gamma\left(\lambda_{i}+1-b+x+i \alpha\right)\right| \\
& \quad \lesssim \sqrt{2 \pi}\left(\alpha+\operatorname{Im}\left(\lambda_{i}\right)\right)^{\operatorname{Re}\left(\lambda_{i}\right)+1-b+x-1 / 2} e^{-(\pi / 2)\left(\alpha+\operatorname{Im}\left(\lambda_{i}\right)\right)} .
\end{aligned}
$$

Therefore,

$$
\begin{gathered}
\left|\Im_{\mathscr{C}_{1}}\right| \leq \sqrt{2 \pi} \int_{c}^{-R}|\Gamma(-x+i \alpha)|\left|\Gamma\left(\lambda_{i}+x-i \alpha\right)\right| \\
\cdot\left|\Gamma\left(\lambda_{i}+1-b+x-i \alpha\right)\right||z|^{-x} e^{\alpha \arg (z)} d x \\
\quad \leqslant \sqrt{2 \pi} \int_{c}^{-R} \alpha^{-x-1 / 2}\left(\alpha+\operatorname{Im}\left(\lambda_{i}\right)\right)^{2 \operatorname{Re}\left(\lambda_{i}\right)+2 x-b} \\
\cdot e^{-\pi \operatorname{Im}\left(\lambda_{i}\right)}|z|^{-x} e^{-\alpha(3 \pi / 2-\arg (z))} d x
\end{gathered}
$$

and, hence,

$$
\lim _{\alpha \longrightarrow \infty}\left|\mathfrak{\Im}_{\lambda_{i}, \mathscr{C}_{1}}\right|=0
$$

provided $\arg (z)<3 \pi / 2$. Almost analogously, it can be shown that

$$
\lim _{\beta \rightarrow \infty}\left|\mathfrak{I}_{\lambda_{i}, \mathscr{C}_{3}}\right|=0
$$

In the last step, we analyse $\mathfrak{\Im}_{\lambda_{i}, \mathscr{C}_{2}}$. We define

$$
\begin{gathered}
\mathfrak{\Im}_{\lambda_{i}, R}=\frac{1}{2 \pi i} \int_{\mathcal{c}-i \infty}^{c+i \infty} \Gamma(R-t) \Gamma\left(\lambda_{i}-R+t\right) \\
\cdot \Gamma\left(\lambda_{i}+1-b-R+t\right)|z|^{R-t} d t
\end{gathered}
$$

Since $t$ is complex valued, we proceed slightly differently than before. Using the Stirling formula again and the well-known identity $|\Gamma(z)| \leq|\Gamma(\operatorname{Re}(z))|$, we obtain

$$
\lim _{R \rightarrow \infty}\left|\mathfrak{\Im}_{R}\right|=0
$$

Finally, we combine

$$
\begin{aligned}
\mathfrak{I} & =\lim _{R \rightarrow \infty} \mathrm{T} \operatorname{diag}\left(\left(\mathfrak{\Im}_{\lambda_{1}, R}, \ldots, \mathfrak{I}_{\lambda_{N}, R}\right)^{\top}\right) \mathrm{T}^{-1}=\mathrm{T} \\
& \cdot \operatorname{diag}\left(\left(\mathfrak{\Im}_{\lambda_{1}, \mathscr{C}_{4}}, \ldots, \mathfrak{\Im}_{\lambda_{N}, \mathscr{C}_{4}}\right)^{\top}\right) \mathrm{T}^{-1}=\frac{1}{2 \pi i} \\
& \cdot \int_{\mathcal{c}-i \infty}^{c+i \infty} \Gamma(-s) \Gamma(\mathrm{L}+s \mathrm{I}) \Gamma(\mathrm{L}+(1-b+s) \mathrm{I}) \\
& \cdot|z|^{-s} d s
\end{aligned}
$$

with the result from the residue theorem.

Let us suppose that $\mathrm{L}$ has the eigenvalue decomposition $\mathrm{L}=\mathrm{T} \Lambda \mathrm{T}^{-1}$. Then, we get

$$
\begin{aligned}
\lim _{c \longrightarrow \infty} e^{-c \mathrm{~L}} & =\mathrm{T} \lim _{c \longrightarrow \infty} \operatorname{diag}\left(\left(e^{-\lambda_{1} c}, \ldots, e^{-\lambda_{N} c}\right)^{\top}\right) \mathrm{T}^{-1} \\
& =\mathrm{T} 0_{N \times N} \mathrm{~T}^{-1}=0_{N \times N},
\end{aligned}
$$

where $0_{N \times N}$ is an $N \times N$ matrix with zero in all entries. Now we take a closer look at the asymptotic behaviour whenever $|z| \longrightarrow \infty$.

Theorem 5. Let $\mathrm{L}$ be a positive stable, diagonalizable matrix. For $|z| \longrightarrow \infty$ the second Kummer function behaves as

$$
\mathrm{U}(\mathrm{L}, b \mathrm{I}, z) \sim|z|^{-\mathrm{L}} .
$$

Proof. We proceed in a similar way to the classical case (see [21, p. 58]). Fix $R>0$. We define a contour integral

$$
\begin{aligned}
\mathfrak{J}_{R} & =\frac{1}{2 \pi i} \\
& \oint_{\mathscr{C}_{R}^{+}} \Gamma(-s) \Gamma(\mathrm{L}+s \mathrm{I}) \Gamma(\mathrm{L}+(1-b+s) \mathrm{I})|z|^{-s} d s
\end{aligned}
$$


where the curve $\mathscr{C}_{R}^{+}$is constructed such that all poles of $\Gamma(-s)$ lie inside and the poles of $\Gamma(\mathrm{L}+s \mathrm{I})$ and $\Gamma(\mathrm{L}+(1-b+s) \mathrm{I})$ outside. This is possible, because examining the residues of the Gamma matrix function, we get

$$
\begin{aligned}
\Gamma(\mathrm{L}+s \mathrm{I})= & \int_{0}^{\infty} e^{-t} t^{\mathrm{L}+s \mathrm{I}-\mathrm{I}} d t \\
= & \int_{0}^{1} \sum_{k=0}^{\infty} t^{\mathrm{L}+(s+k-1) \mathrm{I}} \frac{(-1)^{k}}{k !} d t \\
& +\int_{1}^{\infty} e^{-t} t^{\mathrm{L}+s \mathrm{I}-\mathrm{I}} d t \\
= & \sum_{k=0}^{\infty} \int_{0}^{1} e^{(\mathrm{L}+s \mathrm{I}+(k-1) \mathrm{I}) \ln (t)} \frac{(-1)^{k}}{k !} d t \\
& +\int_{1}^{\infty} e^{-t} t^{\mathrm{L}+s \mathrm{I}-\mathrm{I}} d t \\
= & \sum_{k=0}^{\infty}\left(\int_{-\infty}^{0} e^{(\mathrm{L}+s \mathrm{I}+k \mathrm{I}) u} d u\right) \frac{(-1)^{k}}{k !} \\
& +\int_{1}^{\infty} e^{-t} t^{\mathrm{L}+s \mathrm{I}-\mathrm{I}} d t \\
= & \sum_{k=0}^{\infty} \frac{(-1)^{k}}{k !}(\mathrm{L}+s \mathrm{I}+k \mathrm{I})^{-1} \\
& +\int_{1}^{\infty} e^{-t} t^{\mathrm{L}+s \mathrm{I}-\mathrm{I}} d t .
\end{aligned}
$$

Obviously, $\Gamma(\mathrm{L}+s \mathrm{I})$ has simple poles whenever $\operatorname{det}(\mathrm{L}+(s+$ $k) \mathrm{I})=0$ for $k \in \mathbb{N}$. Hence, $\Gamma(\mathrm{L}+s \mathrm{I})$ is singular if $s=-\lambda_{i}-k$ for all eigenvalues $\lambda_{1}, \ldots, \lambda_{N}$ of L. As $\left|\Im_{R}\right|$ is bounded for large $|z|$, we can write

$$
\begin{aligned}
\mathfrak{I} & =\lim _{R \rightarrow \infty} \mathfrak{I}_{R} \\
& =\left(\sum_{k=0}^{\infty} \Gamma(\mathrm{L}+k \mathrm{I}) \Gamma(1-b-k) \frac{(-|z|)^{-k}}{k !}\right)
\end{aligned}
$$

by the residue theorem. Obviously, for $|z| \longrightarrow \infty$, the integral $\mathfrak{I}$ converges to the unit matrix. On the other hand we already know from Lemma 4 that

$$
\mathfrak{I}=|z|^{\mathrm{L}} \mathrm{U}(\mathrm{L}, b \mathrm{I}, z) \Gamma(\mathrm{L}) \Gamma(\mathrm{L}+(1-b) \mathrm{I}) .
$$

and, hence,

$$
\mathrm{U}(\mathrm{L}, b \mathrm{I}, z)=|z|^{-\mathrm{L}} \Im \Gamma(\mathrm{L})^{-1} \Gamma(\mathrm{L}+(1-b) \mathrm{I})^{-1} .
$$

Moreover, Theorem 5 give us $\lim _{x \rightarrow \infty} \mathrm{U}(\mathrm{L}, b \mathrm{I}, x)=0_{N \times N}$ for $x \in \mathbb{R}$, if $\mathrm{L}$ is positive stable and diagonalizable. Therefore,

$$
\lim _{x \rightarrow \infty} \mathrm{F}_{\mathrm{K}}(x)=\lim _{x \rightarrow \infty} \mathrm{U}\left(-\frac{1}{2} \mathrm{~K}, \frac{1}{2} \mathrm{I}, \frac{x^{2}}{2}\right)=0_{N \times N}
$$

holds for negative stable matrices $\mathrm{K}$.

\section{The Weber Matrix Differential Equation}

The Weber matrix differential equation provides us with an example for the use of parabolic cylinder functions with matrix parameters.

Lemma 6. The general solution of the Weber matrix differential equation

$$
y^{\prime \prime}(x)+\left(\mathrm{K}+\left(\frac{1}{2}-\frac{x^{2}}{4}\right) \mathrm{I}\right) y(x)=0, \quad x \geq 0
$$

has the form

$$
y(x)=\mathrm{D}_{\mathrm{K}}(-x) \overline{\mathrm{c}}_{0}+\mathrm{D}_{\mathrm{K}}(x) \overline{\mathrm{c}}_{1}
$$

with $\overline{\mathrm{c}}_{0}, \overline{\mathrm{c}}_{1} \in \mathbb{R}^{N}$.

Proof. First, we substitute

$$
y(x)=e^{x^{2} / 4} u(x)
$$

into equation (53) and obtain

$$
e^{x^{2} / 4}\left(u^{\prime \prime}(x)+x u^{\prime}(x)+(\mathrm{K}+\mathrm{I}) u(x)\right)=0 .
$$

Multiplying the equality on both sides by $e^{-x^{2} / 4}$, we receive a matrix differential equation that can be solved by a classical power series approach. Then, we take as a solution

$$
u(x)=\sum_{k=0}^{\infty} \overline{\mathrm{c}}_{k} x^{k}, \quad \overline{\mathrm{c}}_{k} \in \mathbb{R}^{N} \forall k \in \mathbb{N} .
$$

If the series is a solution, then the coefficients satisfy the recurrence relation

$$
\overline{\mathrm{c}}_{k+2}=\frac{1}{(k+2)(k+1)}(k \mathrm{I}-(\mathrm{K}+\mathrm{I})) \overline{\mathrm{c}}_{k}, \quad k \geq 0,
$$

separately for even and odd $k$ starting with $\overline{\mathrm{c}}_{0}, \overline{\mathrm{c}}_{1} \in \mathbb{R}^{N}$. First, we obtain the identity

$$
\begin{aligned}
\overline{\mathrm{c}}_{2 k} & =\frac{1}{(2 k) !} \prod_{j=0}^{k-1}(2 j \mathrm{I}-(\mathrm{K}+\mathrm{I})) \overline{\mathrm{c}}_{0}, \\
\overline{\mathrm{c}}_{2 k+1} & =\frac{1}{(2 k+1) !} \prod_{j=0}^{k-1}((2 j+1) \mathrm{I}-(\mathrm{K}+\mathrm{I})) \overline{\mathrm{c}}_{1}
\end{aligned}
$$

by induction and we arrive at

$$
\begin{aligned}
& u(x) \\
& =\overline{\mathrm{c}}_{0}+\sum_{k=1}^{\infty} \frac{1}{(2 k) !} \prod_{j=0}^{k-1}(2 j \mathrm{I}-(\mathrm{K}+\mathrm{I})) x^{2 k} \overline{\mathrm{c}}_{0}+x \overline{\mathrm{c}}_{1} \\
& \quad+\sum_{k=1}^{\infty} \frac{1}{(2 k+1) !} \prod_{j=0}^{k-1}((2 j+1) \mathrm{I}-(\mathrm{K}+\mathrm{I})) x^{2 k+1} \overline{\mathrm{c}}_{1},
\end{aligned}
$$


Using the identity

$$
\begin{gathered}
\prod_{j=0}^{k-1}(2 j \mathrm{I}-(\mathrm{K}+\mathrm{I}))=2^{2 k}\left(-\frac{1}{2}(\mathrm{~K}+\mathrm{I})\right)_{k}, \\
\prod_{j=0}^{k-1}((2 j+1) \mathrm{I}-(\mathrm{K}+\mathrm{I}))=2^{2 k}\left(\frac{1}{2}(\mathrm{I}-(\mathrm{K}+\mathrm{I}))\right)_{k},
\end{gathered}
$$

we finally write

$$
\begin{aligned}
u(x)= & { }_{1} F_{1}\left(-\frac{(\mathrm{K}+\mathrm{I})}{2} ; \frac{1}{2} \mathrm{I} ; \frac{x^{2}}{2}\right) \overline{\mathrm{c}}_{0} \\
& +x_{1} F_{1}\left(\frac{1}{2}(\mathrm{I}-(\mathrm{K}+\mathrm{I})) ; \frac{3}{2} \mathrm{I} ; \frac{x^{2}}{2}\right) \overline{\mathrm{c}}_{1}
\end{aligned}
$$

for $x \geq 0$. Choosing the constants

$$
\begin{aligned}
& \overline{\mathrm{c}}_{0}=\Gamma\left(\frac{1}{2}\right) \Gamma\left(\frac{1}{2}(\mathrm{I}-\mathrm{K})\right)^{-1} \widetilde{\mathrm{c}}_{0}, \\
& \overline{\mathrm{c}}_{1}=\frac{\Gamma(-1 / 2)}{\sqrt{2}} \Gamma\left(-\frac{1}{2} \mathrm{~K}\right)^{-1} \widetilde{\mathrm{c}}_{0},
\end{aligned}
$$

we observe that $\mathrm{F}_{\mathrm{K}}(x)$ solves equation (56). Obviously, $\mathrm{F}_{\mathrm{K}}(-x)$ is another solution and we write

$$
u(x)=\mathrm{F}_{\mathrm{K}}(-x) \widetilde{\mathrm{c}}_{0}+\mathrm{F}_{\mathrm{K}}(x) \widetilde{\mathrm{c}}_{1} .
$$

A proper choice of the constants and a resubstitution give us the desired result.

\section{Conclusions}

Our analysis of the asymptotic behaviour of the second Kummer function with matrix parameters may help to understand better the asymptotic behaviour of other matrix special functions. The method is a generalization of the classical Mellin-Barnes approach that is also used to analyse the properties of other special functions such as the Gauss hypergeometric function. Moreover, matrix special functions have an interesting application in mathematical finance. In overall, our findings might be useful to develop new regime switching models in an Ornstein-Uhlenbeck setting.

\section{Data Availability}

No data were used to support this study.

\section{Conflicts of Interest}

The authors declare that they have no conflicts of interest.

\section{References}

[1] N. N. Lebedev, Special Functions and Their Applications, Prentice-Hall, 1965.

[2] B. Heller, "Special functions and characterizations of probability distributions by zero regression properties," Journal of Multivariate Analysis, vol. 13, no. 3, pp. 473-487, 1983.
[3] M. Abramowitz and I. Stegun, Handbook of Mathematical Functions with Formulas, Graphs, and Mathematical Tables. United States Department of Commerce, National Bureau of Standards, Washington, 1972.

[4] M. Abdalla and A. Bakhet, "Extended Gauss Hypergeometric Matrix Functions," Iranian Journal of Science \& Technology, 2017.

[5] L. Jódar and J. C. Cortés, "Some properties of gamma and beta matrix functions," Applied Mathematics Letters, vol. 11, no. 1, pp. 89-93, 1998.

[6] L. Jódar and J. C. Cortés, "Closed form general solution of the hypergeometric matrix differential equation," Mathematical and Computer Modelling, vol. 32, no. 9, pp. 1017-1028, 2000.

[7] R. A. Rashwan, M. S. Metwally, M. T. Mohamed, and A. Shehata, "Certain Kummer's matrix function of two complex variables under certain differential and integral operators," Thai Journal of Mathematics, vol. 11, no. 3, pp. 725-740, 2013.

[8] R. J. Elliott, T. K. Siu, L. Chan, and J. W. Lau, "Pricing options under a generalized Markov-modulated jump-diffusion model," Stochastic Analysis and Applications, vol. 25, no. 4, pp. 821-843, 2007.

[9] R. J. Elliott and T. K. Siu, "Pricing regime-switching risk in an HJM interest rate environment," Quantitative Finance, vol. 16, no. 12, pp. 1791-1800, 2016.

[10] X. Chen and R. V. Kohn, "Asset price bubbles from heterogeneous beliefs about mean reversion rates," Finance and Stochastics, vol. 15, no. 2, pp. 221-241, 2011.

[11] X. Chen and R. V. Kohn, "Erratum to Asset price bubbles from heterogeneous beliefs about mean reversion rates (Finance Stoch, (2011), 15, (221-241), 10.1007/s00780-010-0124-x)," Finance and Stochastics, vol. 17, no. 1, pp. 225-226, 2013.

[12] L. Jódar and J. C. Cortés, "On the hypergeometric matrix function," in Proceedings of the, vol. 99, pp. 205-217.

[13] L. Jódar, R. Company, and E. Navarro, "Laguerre matrix polynomials and systems of second-order differential equations," Applied Numerical Mathematics, vol. 15, no. 1, pp. 53-63, 1994.

[14] L. Jódar and E. Defez, "A connection between Laguerre's and Hermite's matrix polynomials," Applied Mathematics Letters, vol. 11, no. 1, pp. 13-17, 1998.

[15] R. S. Batahan and A. A. Bathanya, "On generalized laguerre matrix polynomials," Acta Universitatis Sapientiae, Mathematica, vol. 6, no. 2, pp. 121-134, 2014.

[16] E. Defez, L. Jódar, and A. Law, "Jacobi matrix differential equation, polynomial solutions, and their properties," Computers \& Mathematics with Applications, vol. 48, no. 5-6, pp. 789-803, 2004.

[17] L. Jódar, R. Company, and E. Ponsoda, "Orthogonal matrix polynomials and systems of second order differential equations," Differential Equations and Dynamical Systems. An International Journal for Theory, Applications, and Computer Simulations, vol. 3, no. 3, pp. 269-288, 1995.

[18] J. Sastre and L. Jódar, "Asymptotics of the modified bessel and the incomplete gamma matrix functions," Applied Mathematics Letters, vol. 16, no. 6, pp. 815-820, 2003.

[19] Z. M. Kishka, A. Shehata, and M. Abul-Dahab, "On Humbert matrix functions and their properties," Afrika Matematika, vol. 24, no. 4, pp. 615-623, 2013.

[20] A. Shehata, "A new extension of Gegenbauer matrix polynomials and their properties," Bulletin of International Mathematical Virtual Institute, vol. 2, no. 1, pp. 29-42, 2012.

[21] L. J. Slater, Confluent Hypergeometric Functions, Cambridge University Press, 1960. 
[22] R. B. Paris and D. Kaminski, Asymptotics and Mellin-Barnes integrals, vol. 85 of Encyclopedia of Mathematics and its Applications, Cambridge University Press, Cambridge, 2001.

[23] J.-C. Cortés, L. Jódar, F. J. Solís, and R. Ku-Carrillo, "Infinite matrix products and the representation of the matrix gamma function," Abstract and Applied Analysis, vol. 2015, 2015.

[24] H. Buchholz, The Confluent Hypergeometric Function, Springer, Berlin, Heidelberg, New York, 1969.

[25] E. Copson, An introduction to the theory of functions of a complex variable, University Press, Oxford, 1935. 


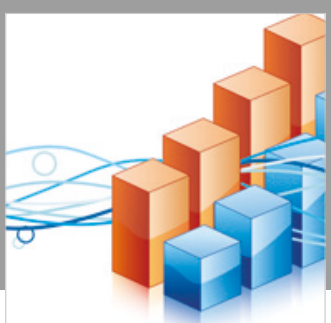

Advances in

Operations Research

\section{-n-m}
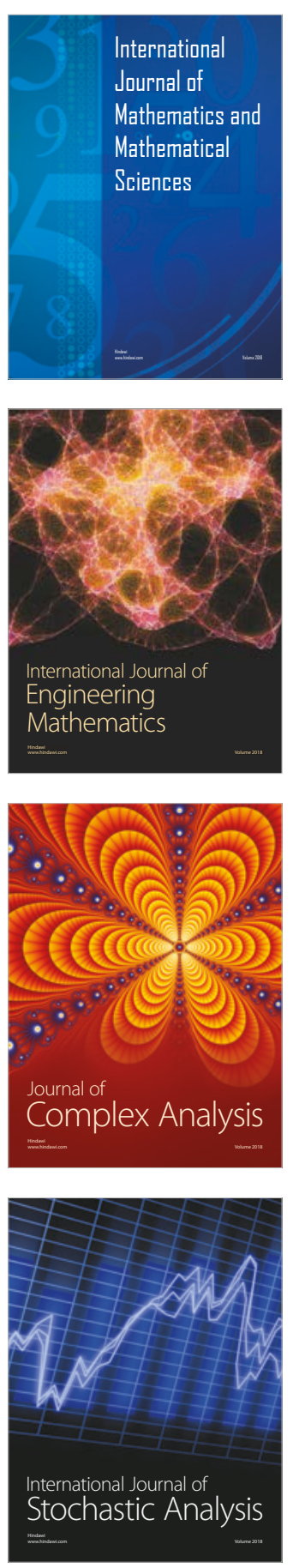
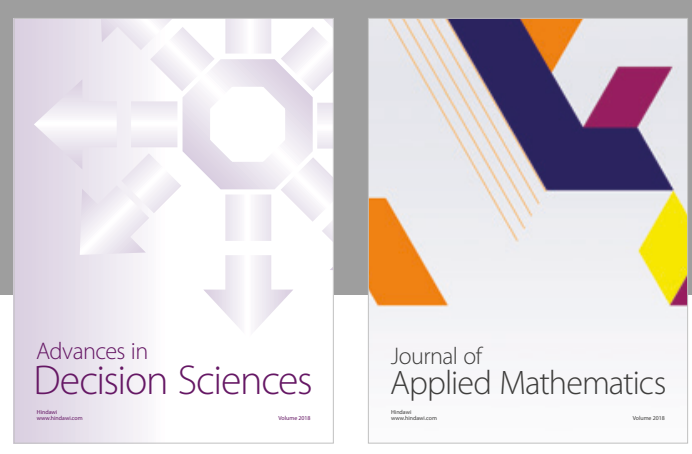

Journal of

Applied Mathematics
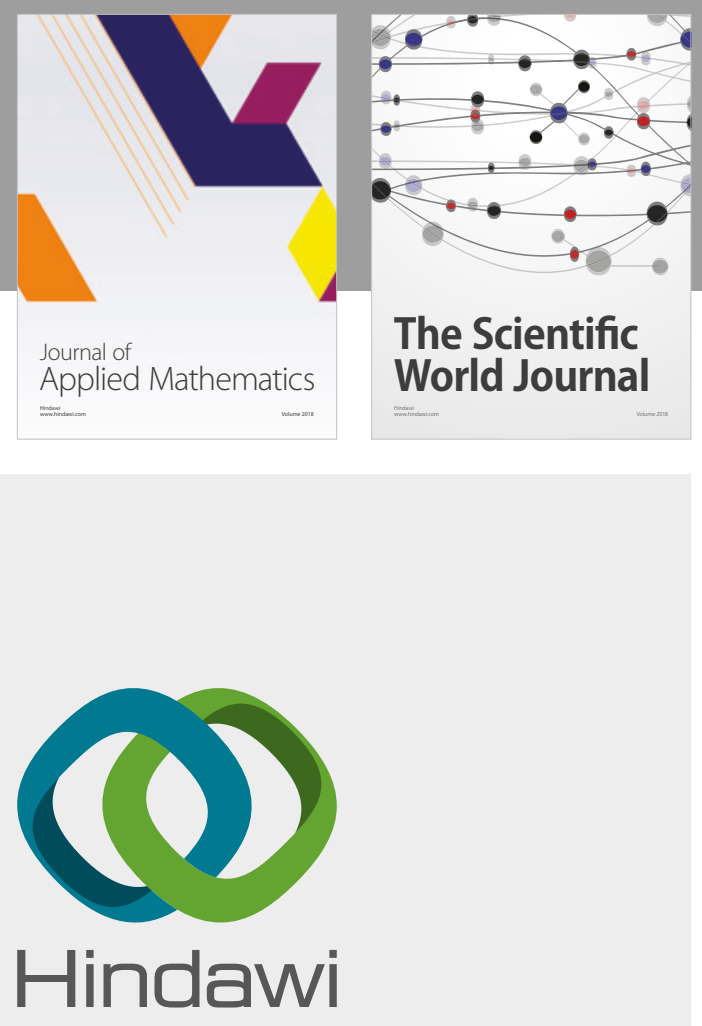

Submit your manuscripts at

www.hindawi.com

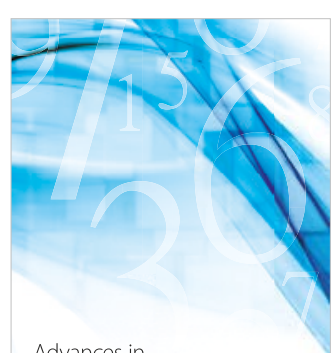

Advances in
Numerical Analysis
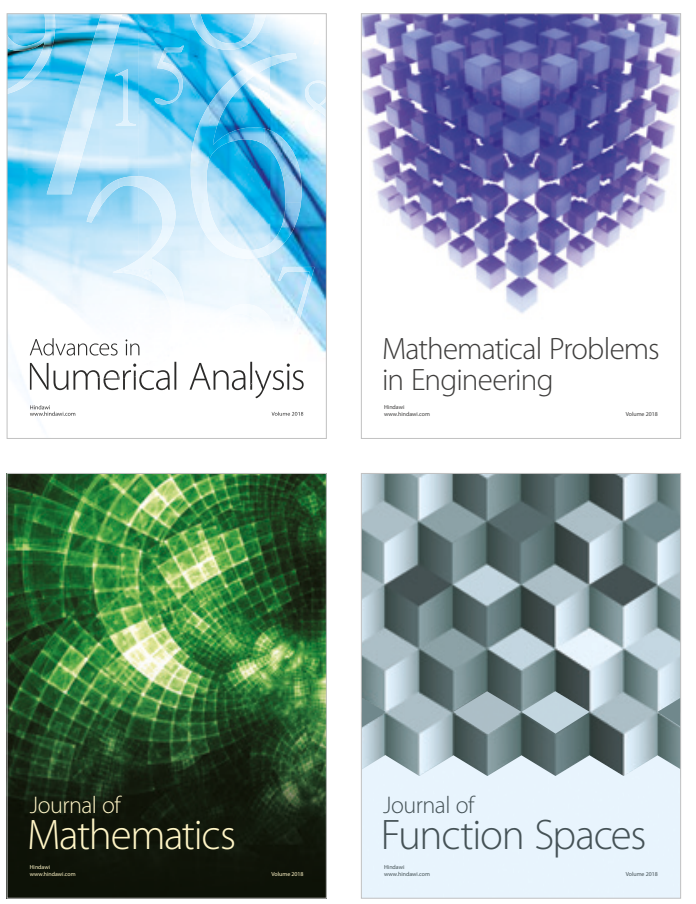

Mathematical Problems in Engineering

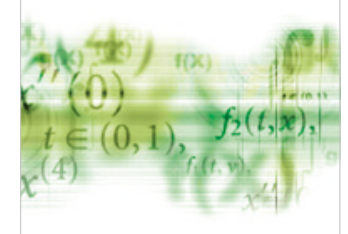

International Journal of

Differential Equations

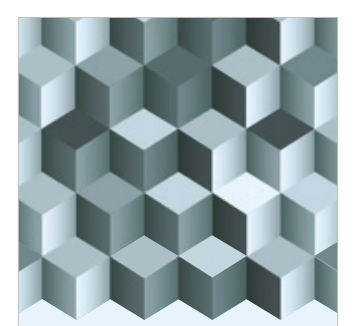

Journal of

Function Spaces

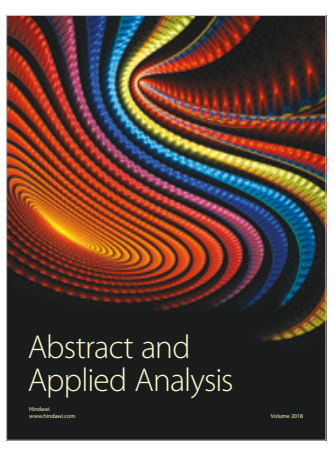

The Scientific

World Journal

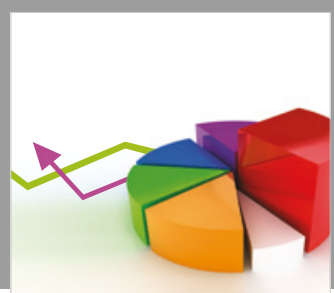

Journal of

Probability and Statistics
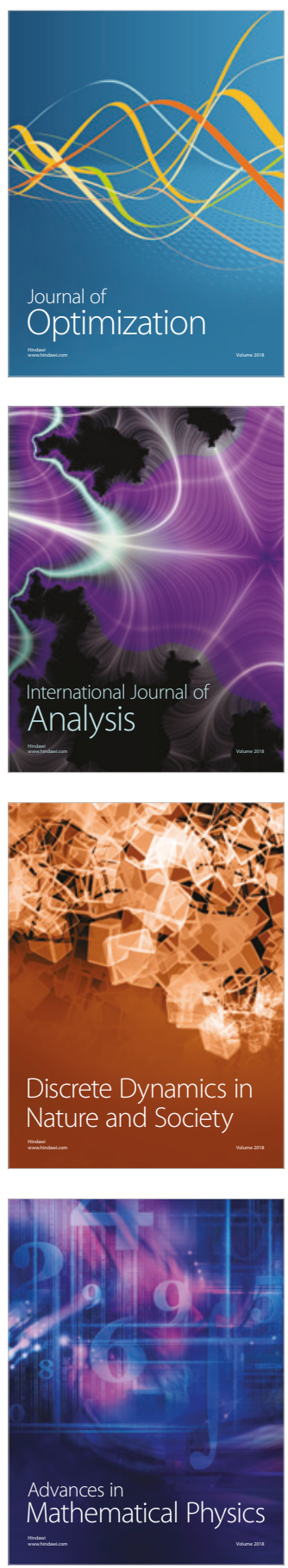